\title{
The Real-Time Temporal and Spatial Diagnostics of Ultrashort High-Power Laser Pulses using an All-Reflective Single-Shot Autocorrelator
}

\author{
Ha-Na Kim ${ }^{1,3}$, Seong Hee Park ${ }^{1 *}$, Kyung Nam Kim ${ }^{1,4}$, Byungheon Han ${ }^{1,5}$, Jae Sung Shin $^{2}$, \\ Kitae Lee ${ }^{1}$, Yong-Ho Cha ${ }^{2}$, Kyu-Ha Jang, Min Yong Jeon ${ }^{3}$, Sergei V. Miginsky ${ }^{1}$, \\ Young Uk Jeong, and Nikolay A. Vinokurov ${ }^{1}$ \\ ${ }^{I}$ Center for Quantum-Beam-based Radiation Research, Korea Atomic Energy Research Institute, \\ Daejeon 305-353, Korea \\ ${ }^{2}$ Quantum Optics Division, Korea Atomic Energy Research Institute, Daejeon 305-353, Korea \\ ${ }^{3}$ Department of Physics, Chungnam National University, Daejeon 305-764, Korea \\ ${ }^{4}$ Department of Physics, Kongju National University, Gongju 314-701, Korea \\ ${ }^{5}$ Department of Physics, Hannam University, Daejeon 306-791, Korea
}

(Received June 19, 2014 : revised July 18, 2014 : accepted July 18, 2014)

\begin{abstract}
An all-reflective, simple noncollinear second harmonic (SH) autocorrelator is described for monitoring the shot-to-shot behavior of ultrashort high-power laser pulses. Two mirrors are used for the dispersion-free splitting of a pulse into two halves. One of the mirrors is able to adjust the delay time and angle between two halves of the laser pulse in a nonlinear crystal. We present the possibility of real-time measurement of the pulse duration, peak intensity (or energy), and the pointing jitters of a laser pulse, by analyzing the spatial profile of the SH autocorrelation signal measured by a CCD camera. The measurement of the shot-to-shot variation of those parameters will be important for the detailed characterization of laser accelerated electrons or protons.
\end{abstract}

\footnotetext{
Keywords : Ultrafast lasers, All-reflective single-shot autocorrelator, Single-shot autocorrelator, Pulse-width measurement

OCIS codes : (140.0140) Lasers and laser optics; (070.4550) Correlators; (140.7090) Ultrafast lasers;

(320.7100) Ultrafast measurements; (190.1900) Diagnostic applications of nonlinear optics
}

\section{INTRODUCTION}

With the development of chirped pulse amplification (CPA) [1], high peak intensity on the order of terawatts in a tabletop femtosecond lasers can be easily accessible in many laboratories, resulting in the rapid developments of related research. In characterizing the physical phenomena in different applications, the real-time measurement of ultrashort high-power laser pulses becomes an issue. For example, in laser-induced acceleration of charged particles the parameters of an ultrashort laser pulse and the plasma it generates are a measure of the quality of laser accelerated particles. The shot-to-shot variation of a charged particle beam especially is an obstacle to using laser accelerators as a radiation source or an injector for other applications, and as a result a detailed characterization involving the jitters of ultrashort laser pulses is inevitable.

The intensity autocorrelation method [2,3], is the simplest and most fundamental approach for measuring the durations of ultrashort pulses. In the 1990, the complete characterization of electric fields with reasonable accuracy was successfully accomplished with frequency-resolved optical gating (FROG) $[4,5]$ and spectral phase interferometry for direct electric field reconstruction (SPIDER) [6, 7]. Those methods can measure both the phase and profile of a laser pulse in time, but with elaborate alignment and iterative phase retrieval algorithm. For intense femtosecond lasers with low repetition rate, a single-shot autocorrelator based on non-collinear second harmonic generation (SHG) $[8,9]$ can measure the pulse duration in real-time, by transferring the

\footnotetext{
*Corresponding author: shpark@kaeri.re.kr

Color versions of one or more of the figures in this paper are available online.
} 
time profile to a spatial profile in a CCD camera. Most modern techniques, including SHG-FROG, GRENOUILLE, and SHG-SPIDER [10], should consider the elongation effect due to the group velocity dispersion in dispersive optics. To suppress the dispersion problem in ultrashort pulse measurements, an all-reflective autocorreleator has been demonstrated [11-13], but these systems, even commercially available ones, are based on the scanning interferometric method using a photodiode detector, which is not proper for real-time characterization.

We suggest instead using a simple, single-shot noncollinear second harmonic ( $\mathrm{SH}$ ) autocorrelator combined with all-reflective split mirrors and a CCD camera, and have performed experiments to investigate the shot-to-shot variation of intense laser pulses, which is an important parameter in characterizing laser-accelerated charged particle beams. In our experiments, the pointing jitter of a laser was simultaneously observed in addition to the pulse duration and peak power. Even though it gives only the pointing jitter in one direction, either horizontal or vertical, it may give information about the pointing stability of a laser-accelerated electron beam. This real-time measurement system can be utilized in any applications using laseraccelerated charged particles (for example, electron or proton therapy) to estimate the beam parameters.

\section{SYSTEM FOR AN ALL-REFLECTIVE SINGLE-SHOT AUTOCORRELATOR}

The basic idea of a single-shot non-collinear SH autocorrelation method is to transform the temporal profile of the original pulse into a spatial profile of a second harmonic pulse in a nonlinear crystal, so that a CCD camera can see the entire temporal trace of a single pulse. The SH signal is generated along the bisector of the incident angle between two split beams entering a nonlinear crystal, satisfying the phase-matching condition for SHG. The important conditions are that the spatial distribution of the original pulse should be smooth and wide enough compared to the pulse length. The diameter of the incident pulse should be greater than three times the longitudinal pulse length.

Assuming that the intensities of two incident pulses are spatially uniform and identical, the SHG at a point $x$ is proportional to $E(t-\tau) E(t+\tau)$, where $\tau$ is the delay time from the peak in intensity. According to the geometry of Fig. 1, $\tau=x n c^{-1} \sin (\alpha / 2)$, where $n$ is the refractive index of the nonlinear crystal and $x$ presents the displacement from the synchronous peak in time.

For a given pulse duration, a smaller incident angle generates a wider spatial profile of the $\mathrm{SH}$ signal. The spatial intensity $S(x)$ of the SH signal at a point $x$ is, then, proportional to the second-order autocorrelation function $G^{(2)}(\tau)$ of the incident pulses, such as:

$$
S(x) \propto \int_{-\infty}^{\infty} I(t-\tau) I(t+\tau) d t=G^{(2)}(2 \tau),
$$

where $I(\mathrm{t})$ is the intensity of the light incident on the non-linear crystal. The constant $n c^{-1} \sin (\alpha / 2)$ for a given set-up can be obtained by measuring the peak position shift $x_{\mathrm{d}}$ of the SH intensity $S(x)$ due to the time delay $t_{\mathrm{d}}$ of one of two pulses. From the geometry (see Fig. 1),

$$
\frac{t_{d}}{x_{d}}=\frac{2 n}{c} \sin \left(\frac{\alpha}{2}\right)
$$

and thus

$$
\Delta \tau_{s}=\frac{t_{d}}{2 x_{d}} \Delta x_{s}
$$

where $\Delta \tau_{\mathrm{s}}$ and $\Delta x_{\mathrm{s}}$ are the pulse duration and spatial width of the SH signals at full width half maximum (FWHM), respectively. The duration of the laser pulse $\Delta t_{\mathrm{P}}$ can be obtained from $\Delta \tau_{\mathrm{s}}$ by multiplying by a constant that depends on the initial laser pulse shape. When the temporal profile is given by the hyperbolic-secant function, the laser pulse shape is written as:

$$
I(t)=I_{0} \operatorname{sech}^{2}\left(\frac{t}{\sigma}\right)
$$

for which the pulse duration at FWHM $\Delta t_{p}=a \sigma$, where $a=2 \ln (1+\sqrt{2})$. The single-shot intensity autocorrelation signal $S(x)$ can be expressed as a function of $\tau$, that is, $S(x) \equiv S(\tau)$ :

$$
S(\tau)=\eta \frac{4 \Delta t_{p}}{a} I_{0}^{2} \times \operatorname{csch}^{2}\left(\frac{2 a \tau}{\Delta t_{p}}\right) \times\left[\frac{2 a \tau}{\Delta t_{p}} \operatorname{coth}\left(\frac{2 a \tau}{\Delta t_{p}}\right)-1\right]
$$

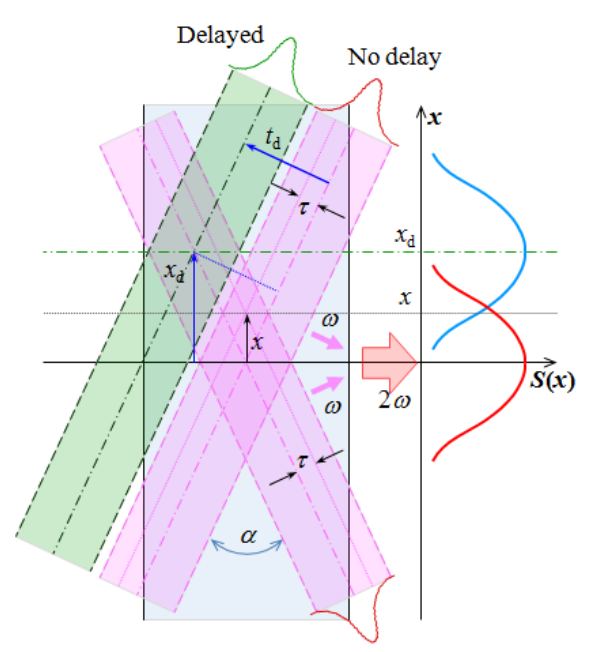

FIG. 1. Schematic of the interaction of two pulses in a nonlinear crystal (ref. 6, Salin et al.). $x_{\mathrm{d}}$ is the shift in peak intensity of the second-harmonic autocorrelation signal due to the time delay $t_{\mathrm{d}}$ of one of two split pulses. 
Here, $\eta$ is a constant related to the conversion factor of second harmonic generation by the non-linear crystal. The FWHM pulse duration of the SH autocorrelation signal is, $\Delta \tau_{s} \cong 1.36 \sigma$, and, therefore, $\Delta t_{p} \cong 1.3 \Delta \tau_{s}$. In the case of a Gaussian pulse shape, $\Delta t_{p}^{G}=\sqrt{2} \Delta \tau_{s}^{G}$. By fitting Eq. (5) to the measured signal, the peak intensity, $I_{0}$, and the FWHM pulse duration, $\Delta t_{\mathrm{p}}$, can be obtained and compared to the measured data. Furthermore, the pulse energy can be calculated by integrating $I(\mathrm{t})$ over time $t$.

$$
U \propto \int_{-\infty}^{\infty} I(t) d t=I_{0} \operatorname{sech}^{2}\left(\frac{t}{\sigma}\right) d t=\frac{2 \Delta t_{p} I_{0}}{a} \propto \Delta t_{p} I_{0}
$$

This can give valuable information in the analysis of the physical phenomena of laser-induced acceleration, since there is no tool to measure the peak power or pulse energy of ultrashort, intense laser pulses in real time. Even though the pulse profile is not an exact secant shape, it is close enough for investigating the contribution of the laser parameters to the generation of laser-accelerated charged particles.

Figure 2 shows a schematic of the all-reflective singleshot autocorrelator. The input beam is spatially split along the y axis into two halves by two rectangular Al-coated mirrors which are separated by a narrow gap $g$ and tilted by a small angle $\theta / 2$ between them. The background-free SH autocorrelation signal is generated along the bisector of the incidence angle $\theta$ between two pulse halves and is measured using a CCD camera behind a band-pass filter. The time scale in the x-axis of the SH image is calibrated with the displacement $x_{\mathrm{d}}$ by varying the optical path length of one half-pulse, corresponding to the delay time $t_{\mathrm{d}}$, using a translation stage to move one mirror.

The nonlinear crystal is a $50 \mu \mathrm{m}$-thick BBO crystal $\left(10 \times 10 \times 0.05 \mathrm{~mm}^{3}\right)$ which is thick enough to measure a pulse duration of $\sim 50$ fs. The angle $\theta$ between two pulse halves is chosen to be $\sim 4^{\circ}$. The time resolution of the

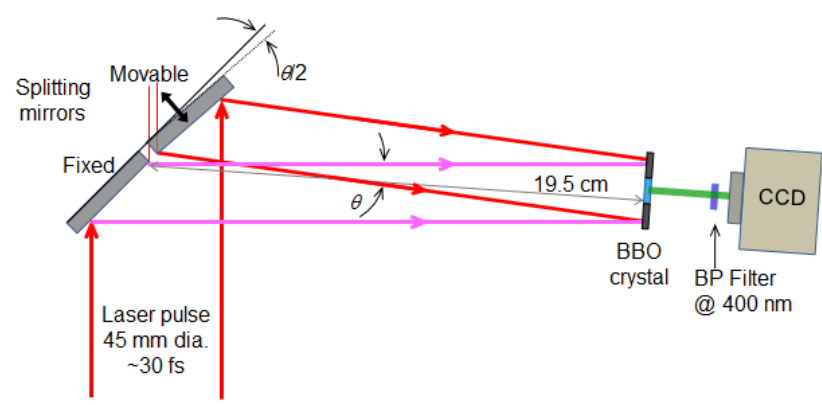

FIG. 2. Schematic of an all-reflective single-shot autocorrelator. One of the splitting mirrors (the upper one) is mounted on a linear translation stage to adjust the delay path length and a BBO crystal $(10 \times 10 \times 0.05 \mathrm{in} \mathrm{mm})$ is located $19.5 \mathrm{~cm}$ from the center of two splitting mirrors, followed by a $400-\mathrm{nm}$ band-pass filter. Due to the mount size, the shortest distance between $\mathrm{BBO}$ crystal and CCD is $3.7 \mathrm{~cm}$. measurement depends on the crossing angle between two incoming beams. For $\theta=4^{\circ}$, the achievable time resolution is around 0.6-1.4 fs per pixel using a CCD camera with 5-12 $\mu \mathrm{m}$ pixels. In our set-up, the time resolution is 0.57 fs/pixel without binning and $1.14 \mathrm{fs} /$ pixel with $2 \times 2$ binning. As the crossing angle is increased, the time resolution is degraded while the background signal due to the near-collinear effect is suppressed.

The KAERI 30 TW laser system, comprised of an oscillator, stretcher, pre-amplifier, and second and third amplifiers, can provide a pulse of $1.1 \mathrm{~J}$ and $300 \mathrm{ps}$ collimated to $\sim 45 \mathrm{~mm}$ in diameter at the entrance of the compression chamber $[14,15]$. The pulse compression using two of $2 \times 2$ inch $^{2}$ diffractive gratings can be optimized by adjusting the distance between the gratings. A compressed beam of $\sim 25 \mathrm{fs}$ in duration and $\sim 45 \mathrm{~mm}$ in size is delivered to the all-reflective single-shot autocorrelator.

Two input beams that are spatially split are identical longitudinally, but not spatially. It is important to use a strongly collimated beam to avoid any modification of pulse duration due to spatial intensity variation. In our case, the contribution due to spatial intensity variation is negligible since the intensity change for the overlapping portion $\left(2 \Delta x_{\mathrm{s}} \sim 280 \mu \mathrm{m}\right.$ in our measurement) is less than $0.05 \%$ for a collimated Gaussian beam of $45 \mathrm{~mm}$ in diameter. According to the geometry in Fig. 2, the overlapping portion in space can be shifted $7 \mathrm{~mm}$ off-center in total, by changing either the location of the $\mathrm{BBO}$ crystal from $19.5 \mathrm{~cm}$ to $30 \mathrm{~cm}$ or the delay path length of one-half-beam. The dependence of pulse duration on spatially overlapping position was hardly observed due to the shot-to-shot variation. The average pulse duration measured was $26.1 \mathrm{fs}$ at 19.5 $\mathrm{cm}$ and $26.3 \mathrm{fs}$ at $30 \mathrm{~cm}$ with a standard deviation of $2-3 \%$, which is within one standard deviation of the shot-toshot variation. To optimize measurement accuracy, one selectes the position where the overlapping portions are symmetric about the center. Any spatial hot spot should be eliminated by carefully matching the main laser and the pump laser beams prior to the experiments.

\section{RESULTS}

Experimental measurements for demonstration were initially performed in the atmosphere after the compressor using a laser beam with pulse energy of $35 \mathrm{~mJ}$. After the performance test of the all-reflective single-shot autocorrelator in the previous section, the pulse compressor was adjusted for the shortest pulse duration before measuring the shot-toshot variation. The photodiode signal measured before the compressor was used as a reference to compare the shot-toshot variation of pulse intensity or energy after the compressor which can give information about shot-to-shot fluctuation of pulse intensity or energy, but not the absolute values. The background due to the SH signal generated only by one half-pulse is subtracted to measure the pulse duration, 


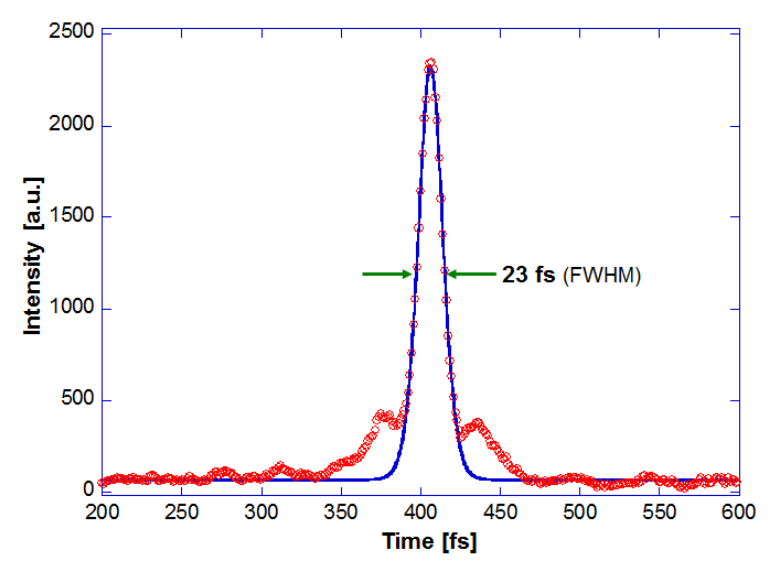

(a) Temporal profile

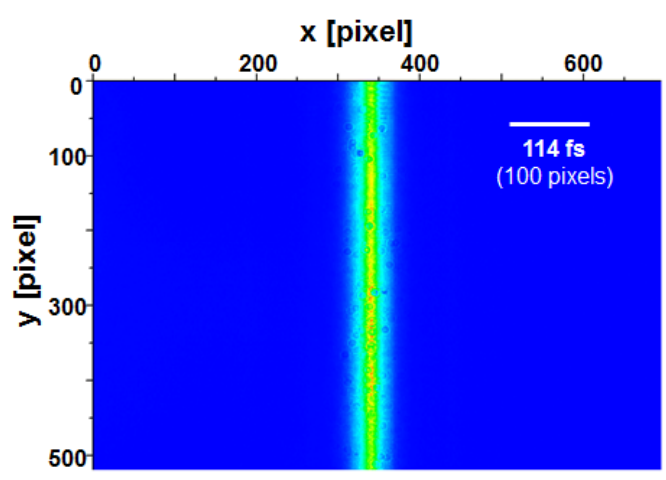

(b) Image of SH signal

FIG. 3. Second harmonic autocorrelation measurements using the single-shot non-collinear autocorrelator. (a) Temporal profile from the measured SH signal (red open circles) and the fitting curve of secant function (blue solid line); (b) Image of SH signal (CCD: $9.3 \times 9.3 \mu \mathrm{m}$ per pixel, $696 \times 520$ arrays).

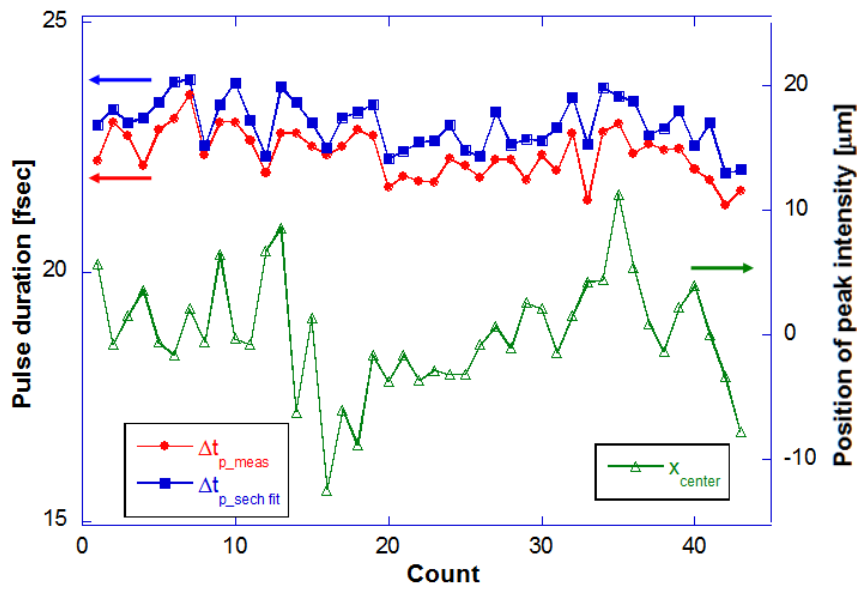

(a) Temporal pulse duration and Position of peak intensity of each SH signal measured

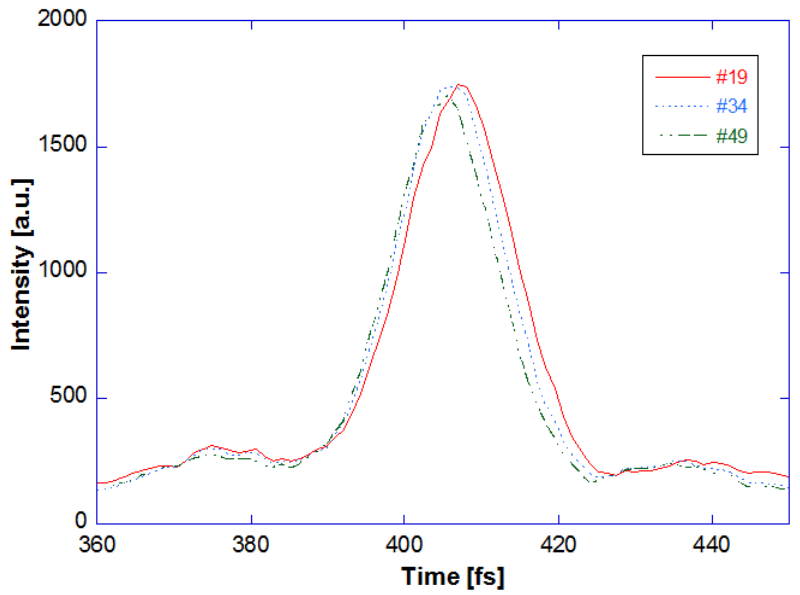

(b) Temporal profiles of three different measurements

FIG. 4. The shot-to-shot variation of laser pulse duration and beam pointing using the single-shot non-collinear autocorrelator. (a) The temporal pulse duration (red solid circles: measured; blue solid squares: calculated from the fitting curve) and the position of peak intensity (green open triangles) for each SH signal measured. (b) The temporal profiles of three different measurements (\#19, \#34, and \#49). The change in peak position corresponds to the pointing shift of the incoming laser beam.

and the temporal pulse profile is assumed to be a sech ${ }^{2}$ function, suitable for the 30-TW femtosecond laser at KAERI.

Figure 3(a) shows the measured $\mathrm{SH}$ signal after background subtraction (red open circles) and the fitting curve (blue solid line). Figure 3(b) is a typical image of the $\mathrm{SH}$ signal captured by the CCD camera. The average pulse duration is $22.4 \mathrm{fs}$ (FWHM) with a standard deviation of $2.2 \%$ as measured from the raw data (red solid circles), and 23.0 fs with $2.1 \%$ standard deviation as obtained from the fitted curve (blue solid squares), as shown in Fig. 4(a). The slight increase in pulse duration for the fitted curves is mostly due to the appearance of side wings in the $\mathrm{SH}$ signals, which could be suppressed by optimizing the alignment of the stretcher and compressor.
It was found that the peak position of the SH signals, as shown in Fig. 4(a) (green open triangles) and Fig. 4(b), changed independently of pulse duration or intensity. The graphs in Fig. 4(b) are three selected SH signals with similar intensities, showing the shot-to-shot variation in pulse duration and peak position. In our splitting configuration, neither the time jitter nor the temporal profile variation can change the peak position of the SH signal; the change seems to be caused by the pointing instability of the main laser. From the geometrical configuration, a pointing angle shift of $1^{\circ}$ will change the coincidence point of two halves in time by $3.4 \mathrm{~mm}$ in space at the nonlinear crystal. The maximum shift observed is $24 \mu \mathrm{m}$, which corresponds to $125 \mu \mathrm{rad}$ after the compressor, and the pointing jitter in 


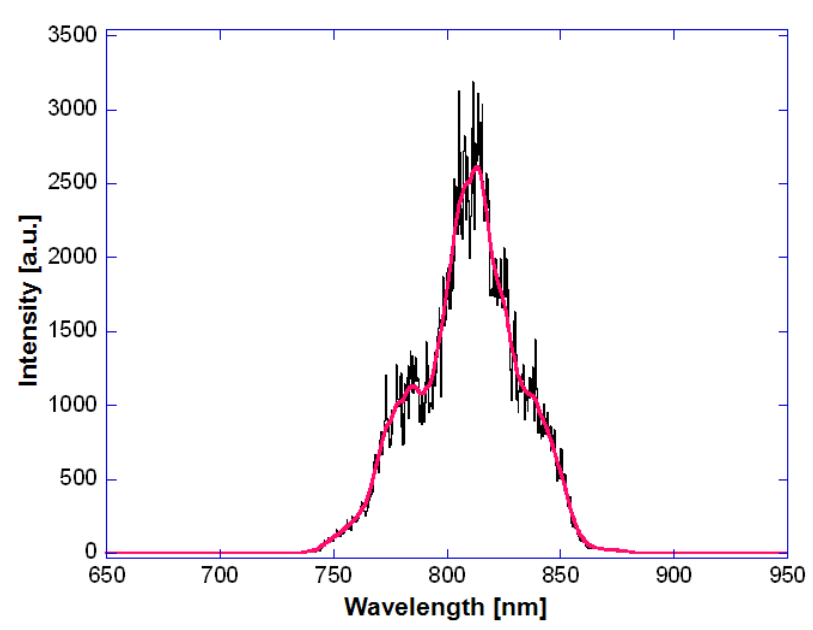

(a)

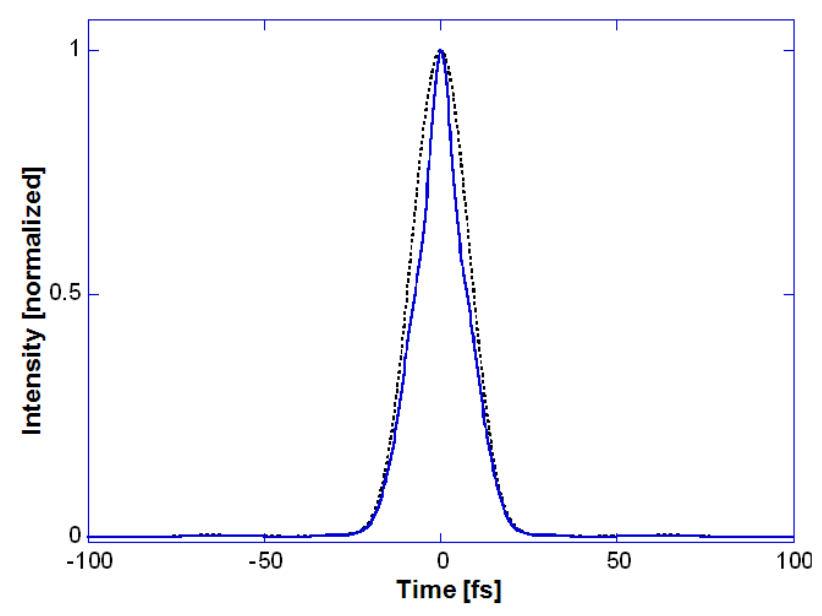

(b)

FIG. 5. The measured spectrum and its Fourier transform (FT): (a) Spectrum measured after the compressor (red solid line: smoothed curve); (b) Fourier transformation of the spectrum assuming no phase difference (dotted line: FT using raw data; solid line: FT using smoothed data).

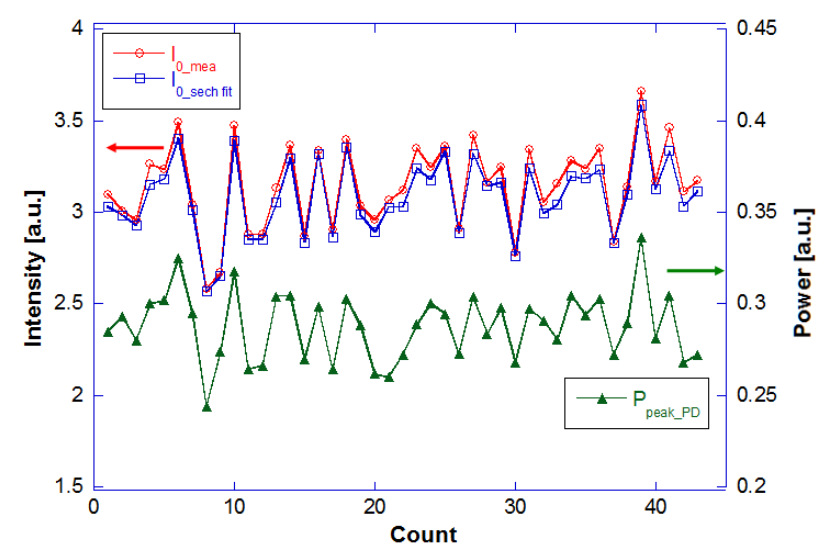

FIG. 6. The shot-to-shot variation of the pulse intensity measured (red open circles) and calculated from the fitting curve (blue open squares) of the SH signals and the peak power of the photodiode signals (green solid triangles). the horizontal direction is about $\pm 24 \mu \mathrm{rad}$ in the standard deviation. The pointing jitter of a laser pulse at the target gives some information about the pointing stability of a laser-accelerated electron beam.

The spectral width $\Delta \lambda$ of the laser pulse after compression was measured to be $\sim 54 \mathrm{~nm}$ (FWHM); see Fig. 5(a). The pulse duration from Fourier transformation (FT) of the spectrum is much shorter than the measured one due to the assumption of zero phase difference, but the shape is closer to the secant function, as shown in Figure 5(b). With the measured pulse duration of $23 \mathrm{fs}$ for a sech ${ }^{2}$ pulse shape, the value of the time-bandwidth product $\Delta t \Delta v$ is 0.576 , indicating that the laser pulse is not bandwidthlimited [16]. The diverging image of the SH signal observed in the CCD located 25 or $30 \mathrm{~cm}$ from the crystal could be evidence of positive chirp [17]. Further investigation and optimization are required.

To demonstrate the possibility of measuring energy or intensity jitter, simultaneously with pulse duration using the single-shot non-collinear $\mathrm{SH}$ autocorrelator, the $\mathrm{SH}$ signals after the compressor were compared to the photodiode signal measured before the compressor. The intensities and energies of the laser pulses from the $\mathrm{SH}$ autocorrelator are obtained by fitting Eq. (5) and Eq. (6) to the SH signals, respectively. The shot-to-shot variations measured by the single-shot autocorrelator and the photodiode are in good agreement, as shown in Fig. 6. This result indicates the possibility of real-time monitoring of both pulse duration and energy (or intensity) using the all-reflective single-shot $\mathrm{SH}$ autocorrelator. The jitters in intensity obtained from the single-shot $\mathrm{SH}$ autocorrelator and the photodiode are $6.9 \%$ and $6.5 \%$ respectively. The jitters in pulse energy increase to $7.8 \%$ and $7.3 \%$ respectively, indicating that the jitters in pulse energy and duration are not correlated.

\section{CONCLUSION}

In summary, an all-reflective single-shot autocorrelator developed to measure the femtosecond pulse-width of a 30 TW high power laser can be a good tool for measuring or monitoring the real time variation of pulse duration and intensity and simultaneously the pointing jitter. For our laser, the shot-to-shot variation in peak intensity, 7\%, dominates the jitter in pulse duration, $2.2 \%$. This result shows that the position jitter has no correlation with pulse duration or intensity. The expected focal-point shift is roughly $\pm 3 \mu \mathrm{m}$ for a focal length of $300 \mathrm{~mm}$ and shifted pointing angle of $\pm 10 \mu \mathrm{rad}$. This may be good enough for proton generation using a metallic target, but not for electron generation via acceleration through the plasma channel of a gas target, resulting in the degradation of the pointing stability of electron beam. The all-reflective singleshot autocorrelator may be used to characterize the performance of laser accelerations. 


\section{ACKNOWLEDGMENT}

This work was partially supported by the World Class Institute(WCI) Program of the National Research Foundation of Korea (NRF) funded by the Ministry of Science, ICT and Future Planning. (NRF Grant Number: WCI 2011-001)

\section{REFERENCES}

1. D. Strikland and G. Mourou, "Compression of amplified chirped optical pulses,” Opt. Commun. 56, 219-221 (1985).

2. M. Maier, W. Kaiser, and J. A. Giordmaine, "Intense light bursts in the stimulated raman effect," Phys. Rev. Lett. 17, 1275-1277 (1966).

3. H. P. Weber, "Method for pulsewidth measurement of ultrashort light pulses generated by phase-locked lasers using nonlinear optics,” J. Appl. Phys. 38, 2231-2234 (1967).

4. R. Trebino and D. J. Kane, "Using phase retrieval to measure the intensity and phase of ultrashort pulses: Frequency-resolved optical gating," J. Opt. Soc. Am. A 10, 1101-1111 (1993).

5. R. Trebino, K. W. DeLong, D. N. Fittinghoff, J. N. Sweetser, M. A. Krumbugel, and B. A. Richman, "Measuring ultrashort laser pulses in the time-frequency domain using frequency-resolved optical grating," Rev. Sci. Instrum. 68, 3277-3295 (1997).

6. C. Iaconis and I. Walmsley, "Spectral phase interferometry for direct electric-field reconstruction of ultrashort optical pulses," Opt. Lett. 23, $792-794$ (1998).

7. C. Iaconis and I. A. Walmsley, "Self-referencing spectral interferometry for measuring ultrashort optical pulses," IEEE J. Quantum Electron. 35, 501-509 (1999).
8. F. Salin, P. Georges, G. Roger, and A. Burn, "Single-shot measurement of a 52-fs pulse," Appl. Opt. 26, 4528-4531 (1987).

9. J. Janszky, G. Corradi, and R. N. Gyuzalian, "On a possibility of analysing the temporal characteristics of short light pulses," Opt. Commun. 23, 293-298 (1977).

10. R. Trebino, "Measuring the seemingly immeasurable," Nat. Photonics 5, 189-192 (2011).

11. I. Z. Kozma, P. Baum, U. Schmidhammer, S. Lochbrunner, and E. Riedle, "Compact autocorrelator for the online measurement of tunable 10 femtosecond pulses," Rev. Sci. Instrs. 75, 2323-2327 (2004).

12. H. Mashiko, A. Suda, and K. Midorikawa, "All-reflective interferometric autocorrelator for the measurement of ultrashort optical pulses," Appl. Phys. B: Lasers Opt. B 76, 525-530 (2003).

13. E. Power, J. Pentland, J. Nees, C. P. Hauri, M. Merano, R. Lopez-Martens, and G. Mourou, "All-reflective high fringe contrast autocorrelator for measurement of ultrabroadband optical pulses," Opt. Lett. 31, 3514-3516 (2006).

14. Y. W. Lee, J. Yi, Y. H. Cha, S. M. Nam, J. M. Han, Y. U. Jeong, Y. J. Rhee, B. C. Lee, and B. D. Yoo, "Development of a 10-TW, 27-fs Ti:sapphire laser system at KAERI," J. Korean Phys. Soc. 49, $412-416$ (2006).

15. J.-Y. Lee, "Study on high effective ion beam acceleration by using the relativistic plasma," Ph.D. Thesis (2011).

16. R. Danielius, A. Stabinis, G. Valiulis, and A. Varanavicius, "Characterization of phase modulated ultrashort pulses using single-shot autocorrelator," Opt. Commun. 105, 67-72 (1994).

17. V. Kabelka and A. V. Masalov, "Determination of spectral width and chirp of ultrashort light pulses by analysis of second harmonic beam divergence," Opt. Commun. 100, 482-486 (1993). 